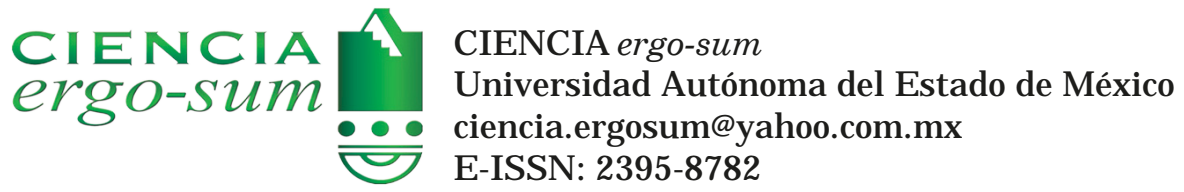

\title{
Serpientes, un legado ancestral en riesgo
}

Martínez-Vaca León, O. I ván; López Medellín, Xavier

Serpientes, un legado ancestral en riesgo

CIENCIA ergo-sum, vol. 26, núm. 2, julio-octubre 2019|e56

Universidad Autónoma del Estado de México, México

Esta obra está bajo una Licencia Creative Commons Atribución-NoComercial-SinDerivar 4.0 Internacional.

Martínez-Vaca León, O. I y López Medellín, X. (2019). Serpientes, un legado ancestral en riesgo. CIE NCIA ergo-sum, 26(2). https://doi.org/10.30878/ces.v26n2a10 


\title{
Serpientes, un legado ancestral en riesgo
}

Snakes, an ancestral legacy at risk

\author{
O. Iván Martínez-Vaca León \\ Universidad Autónoma del Estado de México, México \\ imvleon83@gmail.com \\ Xavier López Medellin \\ Universidad Autónoma del Estado de Morelos, México \\ xlmedellin@uaem.mx
}

Recepción: 07 de junio de 2018

Aprobación: 27 de septiembre de 2018

\section{RESUMEN}

México alberga aproximadamente 393 especies de serpientes, de las cuales 210 son endémicas. Estos reptiles ofrecen servicios ecosistémicos que brindan beneficios a la humanidad como el control de plagas. A pesar de que nuestros ancestros las veneraban, actualmente existe repudio hacia ellas por desconocimiento y falsa información. Por medio de una exhaustiva revisión bibliográfica damos a conocer datos sobre la trascendencia de las serpientes y su diversidad en nuestro país, el desconocimiento, los mitos y el conflicto con el humano. Además, se enfatizan las causas que amenazan su existencia, así como la importancia de la educación ambiental para su conservación. Es nuestra responsabilidad divulgar información confiable que genere percepciones correctas para que se puedan preservar estos reptiles.

PALABRAS ClAVE: serpientes, cultura, diversidad, importancia, conservación, educación ambiental.

\begin{abstract}
Mexico is home to approximately 393 species of snakes, of which 210 only exist within its territory. These reptiles offer ecosystem services that provide benefits to humanity like pest control. Despite the fact that they have been venerated by our ancestors, there is currently a repudiation of them due to the ignorance and false information. Through an exhaustive bibliographic review, we present data regarding the importance of snakes or ophidians in our culture and their diversity in our country. We also mention the benefits they offer, the ignorance and myths towards them and the conflict with humans, emphasizing the causes that threaten their existence, as well as the importance of environmental education for its conservation. It is our responsibility to disclose trustworthy information that generates correct perceptions to preserve these reptiles.
\end{abstract}

KEYWORDS: snakes, culture, diversity, importance, conservation, environmental education.

\section{LA SERPIENTE EN LA MITOLOGÍA MESOAMERICANA}

Desde épocas muy tempranas en la historia de las culturas de Mesoamérica, la serpiente ha representado símbolos místicos, religiosos y militares, entre otros. Los nahuas del centro de México relacionaban a la serpiente con importantes dioses como Quetzalcóatl, Coatlicue y Huitzilopochtli. Quetzalcóatl para ellos, o Kukulkhán para los mayas, es la divinidad que representa la vida y la muerte, Quetzalcóatl conocido como la "Serpiente emplumada” es quizá la leyenda más difundida en América, ya que en muchos sitios arqueológicos de nuestro país existen templos, estelas e imágenes de serpientes con plumas que lo representan (De la Garza, 1984; Martínez y Viñas, 2007; Cano-Contreras y Vargas-Clavijo, 2015).

En el Templo Mayor de Tenochtitlán (actual Ciudad de México), al efectuar trabajos encargados por el virrey en 1790 para remodelar la plaza, se encontró una representación en piedra de la diosa Coatlicue, "la que tiene su falda de serpientes", quien representa la fertilidad, la vida, la muerte y la renovación (figura 1a). También durante estos trabajos descubrieron la "Piedra del Sol" (figura 1b), calendario mexica rodeado por una serpiente de dos cabezas (Xiuhcoatl), que fue el arma de Huitzilopochtli (dios de la guerra), hijo de Coatlicue, nacido en el Cerro de la Serpiente "Coatepec" y cuyo destino fue dirigir al imperio de los guerreros mexicas (De la Garza, 1984; Genis, 2007). 
En Tlaxcala se encuentra la zona arqueológica Cacaxtla. En los frescos que ahí se exhiben se representa una serpiente cornuda con rasgos felinos que se cree está asociada con el cielo y la tierra. El centro ceremonial otomí localizado en Malinalco, Estado de México, es uno de los únicos tres centros ceremoniales tallados en roca que existen en todo el planeta y su forma de serpiente de cascabel representa a una entidad de la tierra; su cabeza y lengua bífida (dividida en dos) es la entrada a este espacio sagrado (figura 1c) (Mendoza, 1977).

Los mayas esculpieron varias estelas y templos donde se muestran algunos de sus gobernantes saliendo del hocico de una serpiente, que representan la posibilidad que tenían estos personajes de acceder a otros planos cósmicos conectados a través de estos animales sagrados (Landrove-Torres, 2014). Los huicholes del noroeste mexicano relacionan a la serpiente emplumada con el planeta Venus y con plantas mágicas sagradas como el peyote (Lophopora williamsi), una cactácea, y la kieri (Solandra brevicalix), pariente del jitomate y el chile. En el Museo de Antropología e Historia de la Ciudad de México hay cuadros de esta cultura que muestran serpientes con astas de venado y líneas ondulantes que simulan lluvia, las cuales representan el encuentro entre el cielo y la tierra y tal vez la dualidad entre la vida y la muerte (Jáuregui, 2002).

Otros pueblos indígenas como los triques y zoques de Oaxaca relacionaban a su diosa madre con la serpiente; de ello existen representaciones de serpientes cornudas asociadas con la tierra, las cuevas y la montaña. Las tribus chichimecas del norte de México relacionaban a la serpiente con el agua y la llamaban Mixcóatl. En Baja California Sur los grupos nómadas cochimí pintaron en cuevas serpientes con cabezas de venado y colas de animales marinos, que se cree simbolizan las estaciones de su calendario y que al ocupar elementos acuáticos, terrestres y celestes podrían reflejar el ciclo del agua o manifestaciones de creación y renovación (Martínez-González et al., 2009).

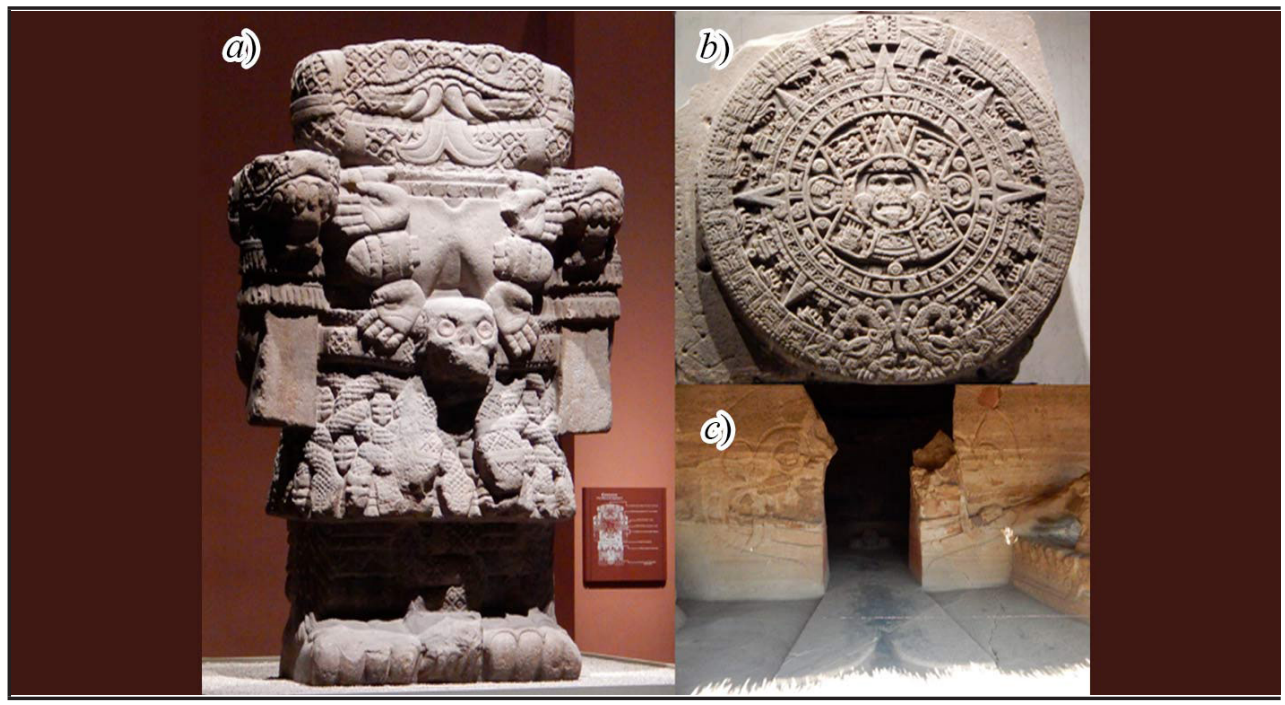

FIGURA 1

Representaciones de serpientes en las culturas prehispánicas del centro de México Fuente: fotografías de Iván Martínez Vaca León.

Nota: a) Coatlicue, Museo Nacional de Antropología e Historia, Ciudad de México; $b$ ) Calendario Azteca, Museo Nacional de Antropología e Historia, Ciudad de México; c) Centro Ceremonial Otomí, Malinalco.

\section{Diversidad de Serpientes en MéXico}

Debido a su conformación geográfica, México es un país privilegiado por contener gran parte de la biodiversidad mundial. Su posición entre dos océanos (Pacífico y Atlántico), sus regiones biogeográficas Neártica y Neotropical, que se identifican cada una por tener diferentes patrones de composición de flora y fauna, además de su relieve y su clima lo hacen un lugar exclusivo que resguarda una gran cantidad de especies únicas o endémicas en el planeta (Jiménez-Sierra et al., 2010). 
En el mundo existen aproximadamente 3701 especies de serpientes (Uetz, 2018) y México, por su parte, alberga a cerca del 11\% (393 especies), de las cuales 53\% (210 especies) son endémicas, es decir, que tienen distribución restringida dentro de su territorio (Flores-Villela y García-Vázquez, 2014). Gran parte de esta diversidad se encuentra distribuida mayormente en tres estados: Oaxaca, Veracruz y Chiapas (tabla 1).

Tabla 1

Estados con mayor diversidad de serpientes

\begin{tabular}{cccc}
\hline Estado & Total de serpientes & Endémicas & Venenosas mortales \\
\hline Oaxaca & 151 & 14 & 26 \\
Veracruz & 138 & 9 & 21 \\
Chiapas & 113 & 6 & 22 \\
\hline
\end{tabular}

Fuente: Morales-Mávil et al., 2011; Johnson et al., 2015; Mata-Silva et al., 2015.

Si bien estos reptiles eran venerados en el pasado como símbolos de dualidad entre la vida y la muerte, también eran considerados medios para acceder a planos cósmicos de acuerdo con la percepción de las culturas mesoamericanas. En la actualidad, sin embargo, la mayoría de la población muestra un repudio y apatía hacia la existencia de estos reptiles por sus características no tan agraciadas, su desconocimiento y en algunos casos por su peligrosidad. A pesar de que las mordeduras de serpientes venenosas representan un problema de salud debido al potente veneno que producen, el cual utilizan para inmovilizar y digerir a su presa, solamente la quinta parte de la biodiversidad en México son mortales para nosotros (78 especies representadas en dos familias: Elapidae y Viperidae con tres y diez géneros respectivamente: Hydrophis, Micrurus, Micruroides y Agkistrodon, Atropoides, Bothrops, Bothriechis, Cerrophidion, Crotalus, Mixcoatlus, Ophryacus, Porthidium y Sistrurus) (Campbell y Lamar, 2004; Flores-Villela y García-Vázquez, 2014).

\section{LAS SERPIENTES Y EL CONFLICTO CON LOS HUMANOS}

El esfuerzo que implica para las serpientes la búsqueda de alimento, y en general el riesgo que representan los mecanismos de defensa de sus presas al capturarlas, ha propiciado en algunos ofidios el desarrollo evolutivo de un sofisticado mecanismo de envenenamiento. Este sistema consta de glándulas especializadas para producir veneno y colmillos modificados para liberarlo (Jackson, 2003).

La energía que necesitan las serpientes para producir el veneno es mucha (McCue, 2006; Morgenstern y King, 2013), por lo que lo usan básicamente para la alimentación: ayuda a paralizar, matar y digerir a la presa (Karaddielle, 1995). No obstante, también es liberado como última opción para la defensa. Cuando hay un encuentro entre humanos y serpientes venenosas, algo probable en zonas rurales, entonces se convierte en un problema de salud pública (Frayre-Torres et al., 2006).

En el ámbito mundial se estima que de todas las mordeduras de serpientes venenosas al año la mitad termina en envenenamientos y sólo 5\% acaba en defunciones (White et al., 2003). Estadísticas del Instituto Mexicano del Seguro Social (IMSS) indican que el número de accidentes ofídicos en promedio por año es de 524 casos, de los cuales $0.8 \%$ (cinco accidentes por mordeduras) terminan en fatalidad (Tay et al., 2002). De todas las serpientes venenosas distribuidas en México, la nauyaca (Bothrops asper), conocida como cuatro narices, sorda, terciopelo, serpiente X, palanca y barba amarilla, es la que ocasiona el mayor número de accidentes. Quintana Roo, Oaxaca y Veracruz son los estados de mayor incidencia (Zertuche, 1981; Frayre-Torres et al., 2006). El gran problema con esta especie se debe a la invasión de su hábitat y a que tiene un excelente camuflaje con las hojas secas del suelo; por esta condición, los pobladores no logran percibirla hasta que la pisan ocasionando la inevitable y defensiva mordedura de esta especie (figura 2). 


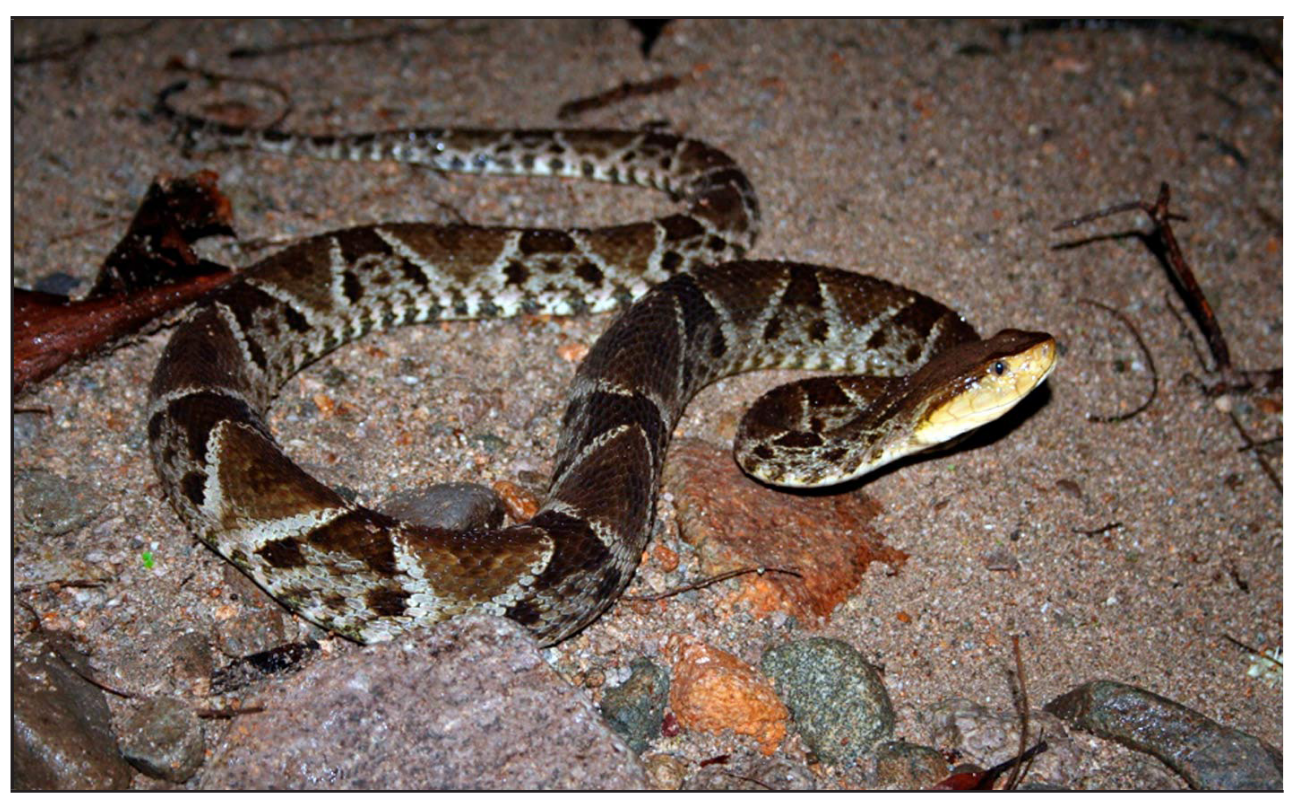

FIGURA 2

Serpiente nauyaca real (Bothrops asper)

Fuente: fotografía de Iván Martínez Vaca León.

Los accidentes ofídicos con las nauyacas pueden representar una causa razonable para temer a esta especie. No obstante, la generalización del miedo hacia cualquier animal con forma de serpiente se ha fomentado por el desconocimiento y los mitos que se construyen sobre las serpientes, llevándolas a su estigmatización e inevitable muerte. Una de estas invenciones consiste en que algunas serpientes succionan los pezones de las mujeres lactantes para alimentarse de la leche materna y le dan su cola al recién nacido para que no se despierte y llore (Jiménez-Guzmán, 1978). Lo anterior es imposible debido a las diferencias entre ellas y los mamíferos: a) como no son mamíferos, no necesitan consumir leche, $b$ ) no tienen labios para succionar los pezones y $c$ ) no tienen mecanismos para digerir la lactosa de la leche.

\section{SERVICIOS ECOSISTÉMICOS QUE OFRECEN LAS SERPIENTES}

Todos los organismos contribuyen directa o indirectamente al funcionamiento de los ecosistemas y dan una gran variedad de servicios que mejoran la calidad de vida del ser humano. En particular, las serpientes son esenciales en la cadena trófica, pues sirven de alimento a otros animales como aves y mamíferos. Por su lugar en esta red alimenticia, son muy importantes para la vida humana debido a que su alimentación está basada en insectos (figura 3), anfibios, reptiles, huevos, polluelos y pequeños mamíferos, ayudando a controlar las poblaciones de especies que dañan importantes cultivos para el ser humano y a evitar que se conviertan en plaga (Valencia-Aguilar et al., 2013; Flores-Villela y García-Vázquez, 2014).

La diversidad de colores y ciertas características llamativas y poco comunes en las serpientes las hacen atractivas para los coleccionistas ya sea para tenerlas de mascotas o simplemente como adornos en sus casas (Fizgerald et al., 2004). Esta actividad llega a generar negocios legales, pero también ilegales, donde se manejan grandes cantidades de dinero y especies en el mundo (Alvarado et al., 2017), y seguramente sin considerar las condiciones de conservación de sus poblaciones. La piel de algunas serpientes también es muy utilizada en la fabricación de utensilios de uso diario como carteras, botas, chamarras, bolsas, entre otros objetos, incluso algunos músicos buscan estas pieles para utilizarlos en los arcos de sus instrumentos de cuerda como el violín y el violonchelo sin considerar su origen (figura 4). 


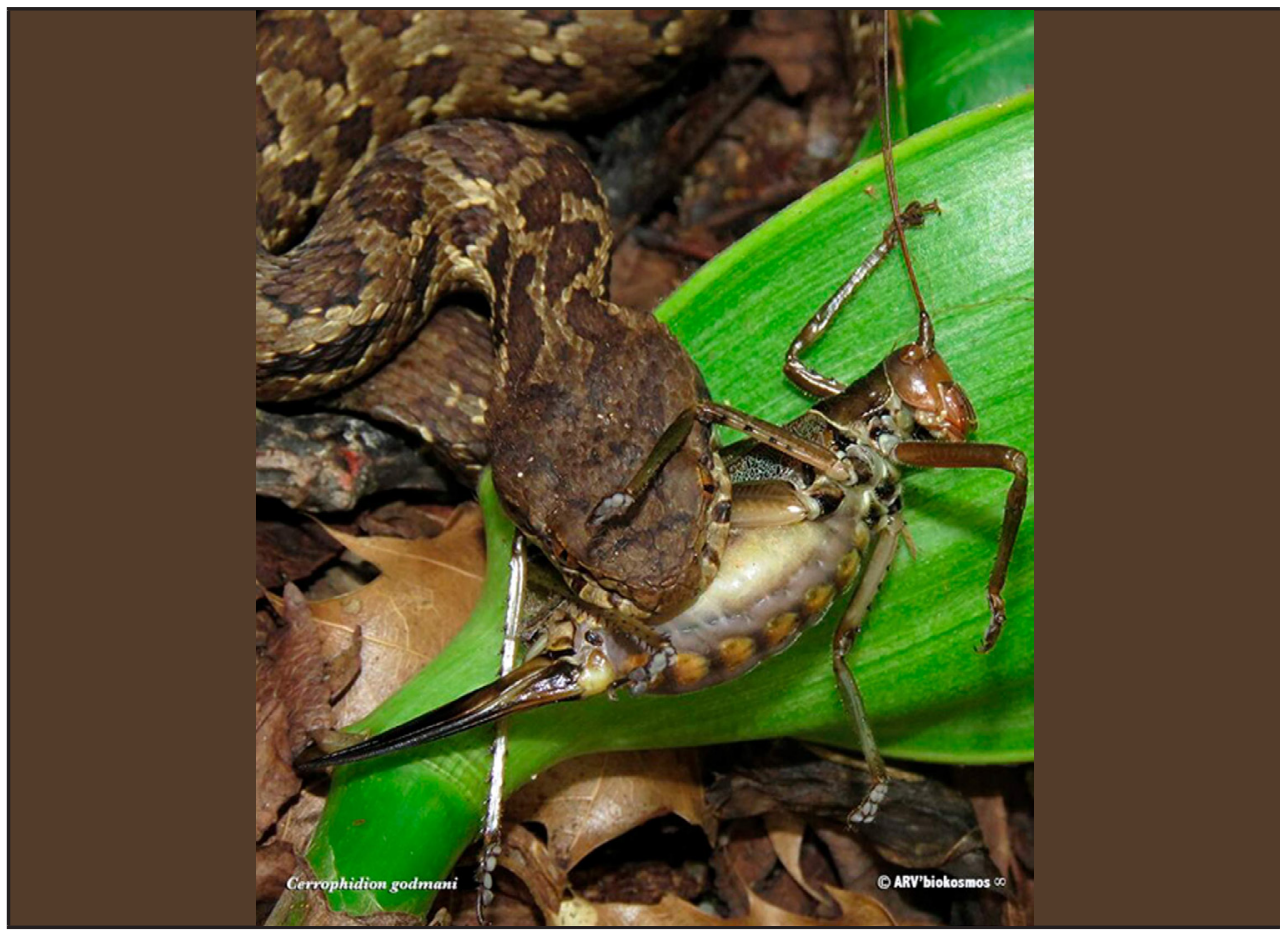

FIGURA 3

Serpiente nauyaca de montaña (Cerrophidion godmani) capturando un saltamontes Fuente: fotografía de Antonio Ramírez Velázquez.

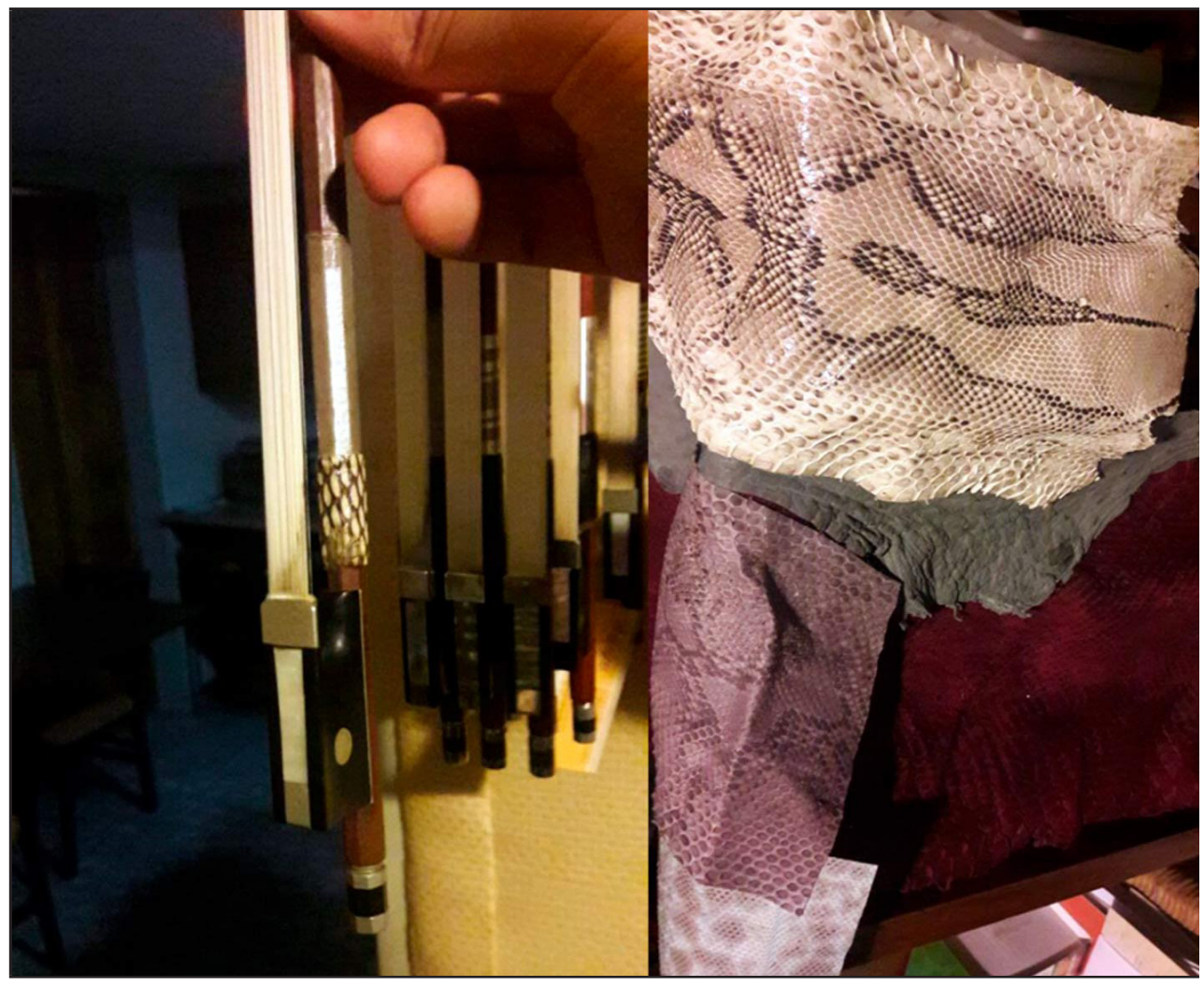

FIGURA 4

Piel de serpiente utilizada para recubrir instrumentos musicales Fuente: fotografías de Xavier López Medellín. 
En varias regiones, sobre todo en comunidades rurales e indígenas en México y el resto de América Latina, las serpientes son valoradas por representar una importante fuente de proteínas, por lo que su cacería es una actividad significativa para la subsistencia de estas personas (Valencia-Aguilar et al., 2013). Por otro lado, el conocimiento que muchos ancianos y curanderos tienen sobre estos reptiles es de gran importancia en la medicina tradicional, ya que los usan para tratar padecimientos como asma, reumatismo, hemorragias, artritis, osteoporosis o tumores, por lo que es posible encontrarlos a la venta en mercados locales (Gómez-Álvarez et al., 2005). En estos sitios la carne de serpiente de cascabel es bastante cotizada debido a sus supuestas propiedades curativas contra el cáncer y diabetes (Gómez y Pacheco, 2010). Las investigaciones muestran que las verdaderas propiedades terapéuticas que las serpientes ofrecen resultan del funcionamiento de las toxinas de sus venenos (Mackessy, 2010), mas no de su carne, y ponen de manifiesto sus habilidades anticancerígenas, antitrombosis (evita la formación de coágulos en las arterias), coagulantes, hipotensivas (disminuye la presión en las arterias) mediante la fabricación de diferentes fármacos como por ejemplo Protac ${ }^{\oplus}$, Defibrasa ${ }^{\oplus}$, Arvin $^{\oplus}$, Captopril ${ }^{\circ}$, entre otros (Koh y Manjunatha, 2011; Rivas, 2014), para los cuales se utilizan serpientes criadas en cautiverio.

Por ello, aunque las serpientes venenosas pueden representar un problema de salud pública, por supuesto mucho menor que otras circunstancias que en la actualidad amenazan nuestra integridad física, psicológica y emocional, su importancia médica les confiere una relevancia que va más allá de lo biológico, ecológico, económico y cultural. Desafortunadamente, los servicios que estos reptiles brindan a la humanidad no son conocidos, reconocidos y valorados; por el contrario, el temor e ignorancia hacia ellos han propiciado su muerte provocando que sus poblaciones disminuyan de manera drástica, lo cual pone en riesgo su existencia $y$, por consecuencia, la potencial ayuda que nos brindan.

\section{Amenazas a la conservación de las Serpientes}

Durante los últimos sesenta años, ciertos factores como los avances tecnológicos, el desarrollo industrial, el crecimiento poblacional y la falta de empleo han ocasionado en México cambios que han transformado negativamente sus ecosistemas y dañado su biodiversidad (Challenger y Dirzo, 2009). Al respecto, existen varias actividades en común que han afectado y disminuido las poblaciones animales, incluyendo las de las serpientes.

La fragmentación, transformación y destrucción del hábitat son las principales amenazas para cualquier organismo (Morales-Mávil et al., 2011), ya que aíslan sus poblaciones o las destruyen completamente. Estas actividades afectan la continuidad de sus hábitats para la búsqueda de recursos y su viabilidad genética, alteran sus fuentes de recursos alimenticios, de resguardo y de reproducción, los remueven en su totalidad de su medio de subsistencia y en el peor de los casos ocasionan su muerte (Ejeta y Bekele, 2017).

Por si fuera poco, si los organismos sobrevivieran a tales actividades y se llegaran adaptar a las nuevas condiciones de hábitat, la construcción de carreteras, la urbanización y prácticas agropecuarias terminarían por complementar las amenazas a su supervivencia (Morales-Mávil et al., 2011) (figura 5). Sumado a lo anterior, existen otros factores colaterales como el calentamiento global, la contaminación de cuerpos de agua, de ecosistemas y las enfermedades ocasionadas por estas condiciones que modifican su distribución, reproducción, crecimiento y movimiento propiciando la reducción de sus poblaciones (Aubret y Shine, 2010; Ejeta y Bekele, 2017).

Otra de las problemáticas que agravan y presionan la supervivencia de las serpientes es el comercio ilegal (Alvarado et al., 2017). Esta actividad ilícita surge como consecuencia de la falta de fuentes de empleo o la mala remuneración de los que existen, puesto que representan una opción cómoda de vivir con buenas ganancias. A pesar de que los coleccionistas en general mantienen en buenas condiciones los ejemplares adquiridos, sobre todo por su elevado costo, la viabilidad de las poblaciones silvestres disminuye al reducirse el número de sus individuos en la naturaleza a causa del tráfico ilícito (Alvarado et al., 2017). 
Si además de todos estos factores que amenazan la existencia de las serpientes, agregamos la apatía y repudio por su naturaleza, el temor, el desconocimiento, la ignorancia y los falsos mitos creados sobre estos reptiles, es indudable y prioritaria la necesidad de ayudar y contribuir a su conservación sobre todo si consideramos que la mayor parte de las amenazas y presiones para su supervivencia son ejercidas por nuestra especie.

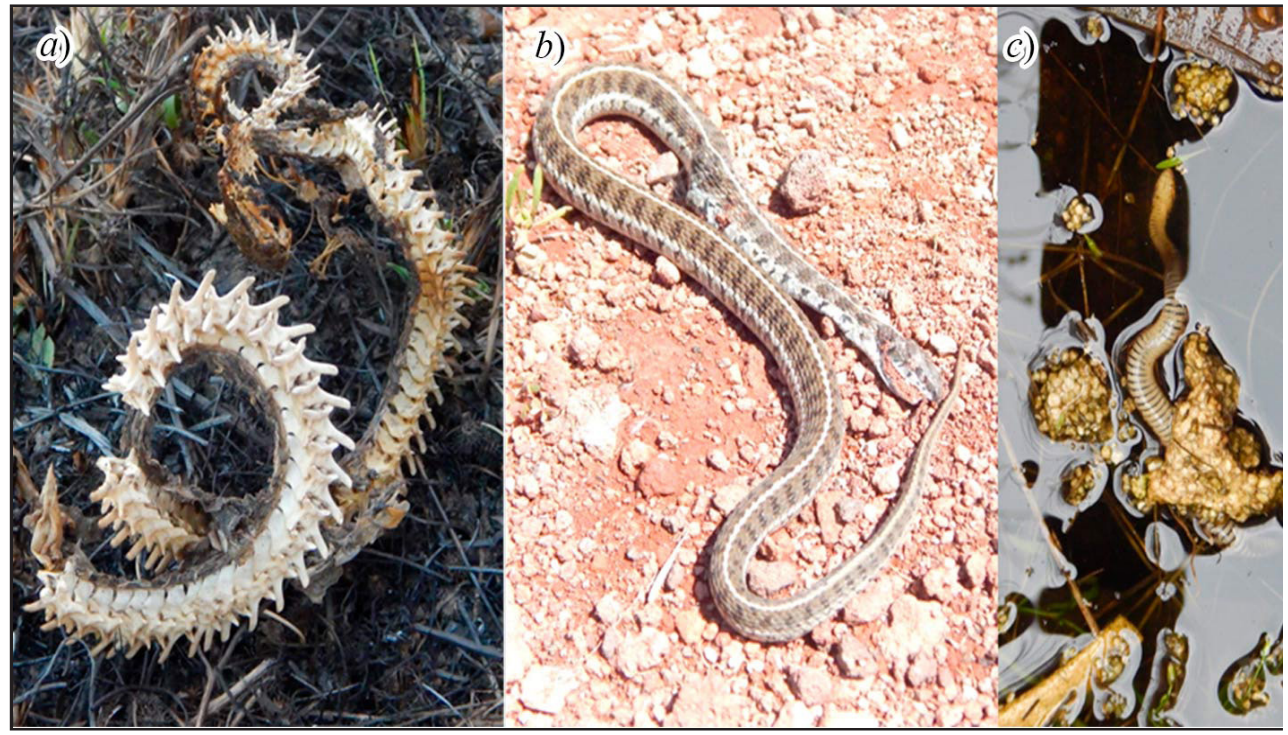

FIGURA 5

Ejemplo de casos de muerte de serpientes por causas humanas

Fuente: fotografías de Iván Martínez Vaca León.

Nota: a) serpiente de cascabel calcinada por prácticas de quema en zona de cultivo; $b$ ) serpiente de agua atropellada cerca de carretera; $c$ ) serpiente ahogada en un registro de drenaje.

\section{Educación ambiental y conservación de las serpientes en MéXico}

La gente de las ciudades considera comúnmente que todas las serpientes son venenosas y mortales o cree también que si una persona se topa con una de ellas, ésta de inmediato la morderá. De ninguna manera esto es así; por el contrario, en la mayoría de los casos las serpientes se asustan con cualquier movimiento brusco y prefieren huir en cuanto detectan la presencia humana.

Es necesario conservar a las serpientes tanto por su importancia como por el riesgo que tienen de desaparecer (Valencia-Aguilar et al., 2013). Una estrategia en México que contribuye a la protección de las especies de plantas y animales es la creación de áreas naturales protegidas (territorios con biodiversidad decretados como prioritarios para la conservación). Sin embargo, solamente 61\% de los reptiles en México se encuentran en alguna área natural protegida (Paredes-García et al., 2011). En un estudio se determinó que de las 34 especies de serpientes de cascabel que viven en México, 30 están en alguno de estos sitios de protección; estos datos dejan un vacío interesante por explorar y evaluar para el resto de las serpientes venenosas y no venenosas de nuestro país (Paredes-García et al., 2011).

Una acción necesaria para convencer a las personas sobre la importancia que tienen las serpientes es la educación ambiental, la cual busca que la sociedad ayude a proteger y mejorar el ambiente (figura 6). Esta educación se puede implementar en las escuelas, zoológicos, museos o centros de investigación. Un ejemplo de lo anterior es la que realiza el Zoológico Miguel Álvarez del Toro en Chiapas, donde además de exhibir especies de serpientes endémicas de la región, dan explicaciones sobre ellas, hablan de su función en el ecosistema, de las amenazas a su conservación y además despejan mitos y creencias que merman el conocimiento y la percepción de las personas. 


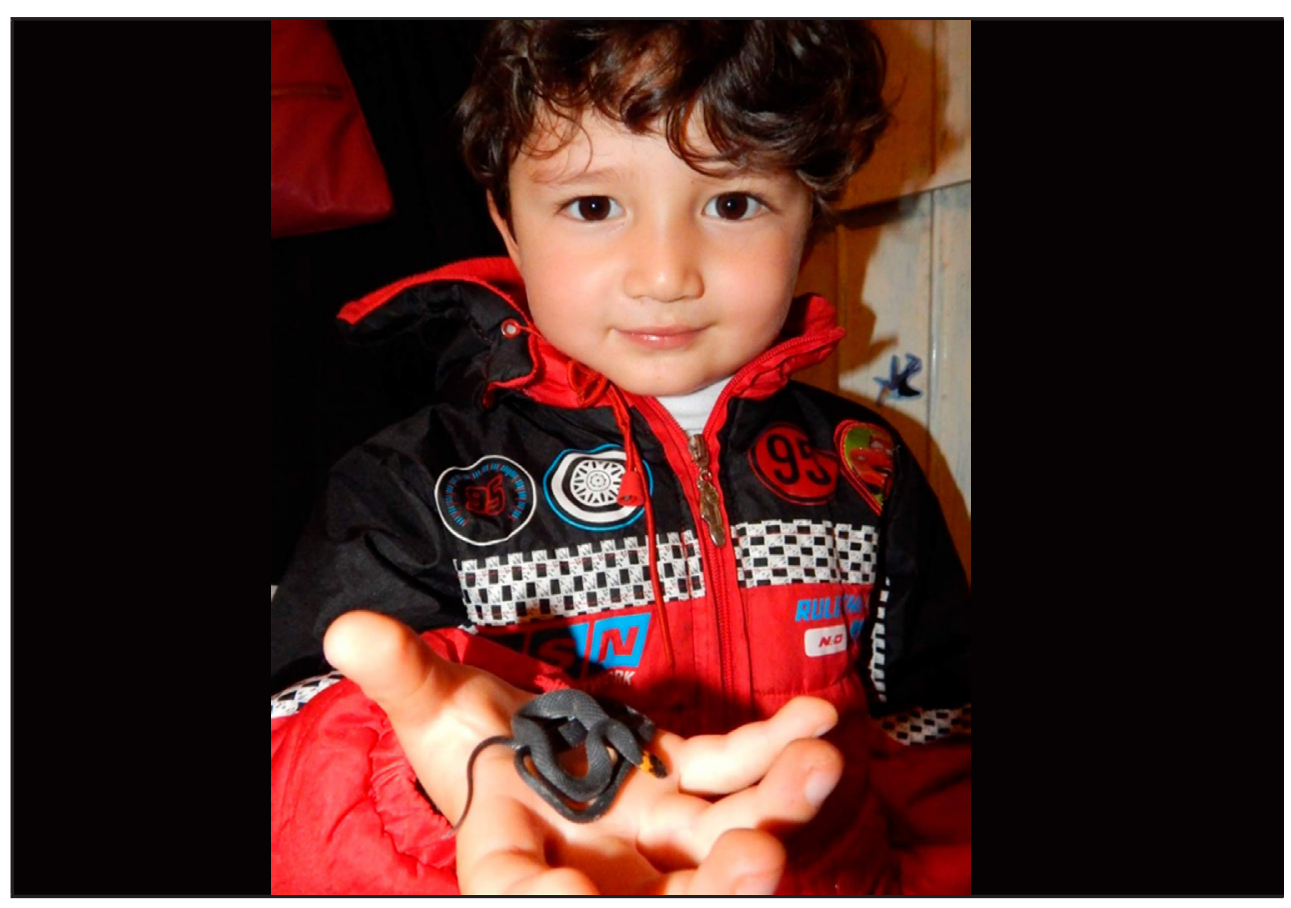

FIGURA 6

Niño de 3 años sosteniendo sin prejuicios ni temor una culebra de collar o de cafetal (Ninia diademata) Fuente: fotografía de Iván Martínez Vaca León.

\section{El FUTURo de LAS SERPIENTES}

Las serpientes han sido veneradas y consideradas animales sagrados por nuestros ancestros desde hace siglos. Si bien en aquellos tiempos eran respetadas por su relevancia en lo espiritual, la evolución de las sociedades en sus intereses enfocados hacia lo material y monetario como sinónimo de poder ha dejado atrás el equilibrio ecológico en el que el respeto por la vida era la base de la armonía. Actualmente, estos reptiles son estigmatizados por muchos sectores de la sociedad, pues les atribuyen poderes malignos que crean percepciones erróneas de su existencia. Por otro lado, el desconocimiento de su biología y de sus aspectos ecológicos ha ocasionado que se propague información equivocada. Además, la naturaleza depredadora de las serpientes, que en un pequeño número de especies está complementada por adaptaciones evolutivas como la producción de veneno, aumenta la apatía y el repudio hacia ellas como consecuencia de los accidentes ofídicos ocasionados de manera directa por problemas ambientales como la pérdida de su hábitat a causa de las diversas actividades humanas. Si bien el futuro de estos seres vivos es incierto y desalentador, es deber y responsabilidad de los profesionales comprometidos con el estudio de la vida y expertos en el tema transmitir adecuadamente el conocimiento con la finalidad de educar a las personas sobre estos reptiles, así como de sus servicios ecosistémicos y los beneficios que brindan a la humanidad.

\section{Conclusiones}

Las serpientes nos ofrecen servicios ecológicos, biológicos, económicos, medicinales y culturales que benefician nuestra calidad de vida. Sin embargo, la fragmentación, transformación y destrucción del hábitat, por la urbanización y prácticas agropecuarias, el calentamiento global, la contaminación de cuerpos de agua, de ecosistemas, las enfermedades, el comercio ilegal, la apatía, repudio, temor, desconocimiento y la información incorrecta son causas que amenazan su existencia. Aunque la creación de áreas naturales protegidas es una buena estrategia para 
su resguardo, es nuestra responsabilidad como sociedad preservar este grupo de vertebrados que forma parte de nuestro patrimonio nacional y cultural. Es fundamental aumentar el esfuerzo para divulgar el conocimiento sobre estos reptiles con el objetivo de que las personas tengan información adecuada que les genere una percepción correcta, de respeto y valoración que estos animales merecen. Asimismo, es prioritario crear conciencia a través de la educación ambiental, así como enfatizar en estrategias para su conservación.

\section{Agradecimientos}

Se agradece a Antonio Ramírez Velázquez, curador de la colección de reptiles y anfibios del Zoológico Miguel Álvarez del Toro (ZOOMAT) por su autorización para reproducir la fotografía de Cerrophidion godmani capturando un saltamontes. Agradecemos a su vez a los árbitros de la revista por enriquecer el documento con sus comentarios. A Sebastian Kwapisz por su aportación del uso de pieles para los arcos de instrumentos de cuerdas.

\section{ReFERENCIAS}

Alvarado, E., Estrada, A. M. y Melgoza, A. (2017). Tráfico de animales. Comercio ilegal en México. México: Ediciones B México.

Aubret, F., \& Shine, R. (2010). Thermal plasticity in young snakes: how will climate change affect the thermoregulatory tactics of ectotherms? The Journal of Experimental Biology, 213, 242-248.

Campbell, J. A., \& Lamar, W. W. (2004). The venomous reptiles of the western Hemisphere Volume 1. New York: Cornell University Press.

Cano-Contreras, E. y Vargas-Clavijo, M. (2015). Culebra guardacaminos. ¿Por qué me quieres picá?: representaciones culturales alrededor de la mordedura de serpiente entre colombianos y mexicanos. Ouricuri, 5(1), 145-167.

Challenger, A. y Dirzo, R. (2009). Factores de cambio y estado de la biodiversidad, en Capital natural de México, vol. II: estado de conservación y tendencias de cambio. México: CONABIO.

De la Garza, M. (1984). El universo sagrado de la serpiente entre los mayas. UNAM. Instituto de Investigaciones Filológicas.

Ejeta, A. G., \& Bekele, G. (2017). Threats to biodiversity and the role of conservation biology for future sustainability: A review. International Journal of Research-Granthaalayah, 5, 238-242.

Fitzgerald, L. A., Painter, C. W., Reuter, A., \& Hoover, C. (2004). Collection, trade, and regulation of reptiles and amphibians of the Chihuahuan Desert Ecoregion. Traffic North America-WWF. Washington D. C.

Flores-Villela, O. y García-Vázquez, U. O. (2014). Biodiversidad de reptiles en México. Revista Mexicana de Biodiversidad, 85, 467-475.

Frayre-Torres, M. J., Sevilla-Godínez, E., Orozco-Valerio, M. de J., Armas, J. y Celis, A. (2006). Mortalidad por contacto traumático con serpiente y lagarto venenosos. México, 1979-2003. Gaceta Médica de México, $142(3), 209-213$.

Genis, J. (2007). El monolito de la Coatlicue. Revista Trabajadores, 58, $49-51$.

Gómez-Álvarez, G., Reyes-Gómez, S. R., Teutli-Solano, C. y Valadez-Azúa, R. (2005). La medicina tradicional prehispánica, vertebrados terrestres y productos medicinales de tres mercados del Valle de México. Etnobiología, 5, 86-98.

Gómez, A. G. y Pacheco, C. N. (2010). Uso medicinal de serpientes comercializadas en dos mercados de la ciudad de México. Etnobiología, 8, 51-58.

Jackson, K. (2003). The evolution of venom delivery systems in snakes. Zoological Journal of the Linnean Society, 137, 337-354. 
Jáuregui, J. (2002). La serpiente emplumada entre los coras y huicholes. Arqueología Mexicana, 9(53), 64-65. Jiménez-Guzmán, A. (1978). Las serpientes y sus leyendas. II Congreso Nacional de Zoología. Facultad de Ciencias Biológicas, Universidad Autónoma de Nuevo León, 6-9 de diciembre. 1, 284-297.

Jiménez-Sierra, C., Torres-Orozco B. R. y Corcuera, M. P. (2010). Biodiversidad: una alerta. Casa Del Tiempo, 3(36), 9-16.

Johnson, J. D., Mata-Silva, V., García-Padilla, E., \& Wilson, L. D. (2015). The herpetofauna of Chiapas, Mexico: composition, distribution, and conservation. Mesoamerican Herpetology, 2, 271-329.

Karaddielle, L. (1995). Animal toxins. British Journal of Anesthesia, 74(3), 319-327.

Koh, C. Y., \& Manjunatha, K. R. (2011). From snake venoms toxins to therapeutics. Cardiovascular examples. Toxicon, 59, 497-506.

Landrove-Torres, H. C. (2014). Una “Serpiente de Visión” en Yaxchilán. ¿Encarnación chamánica maya? El tlacuache, 604, 2-4.

Mackessy, S. P. (2010). The handbook of venoms and toxins of reptiles. USA: CRC Press.

Martínez, R. y Viñas, R. (2007). Palabras e imágenes de la vieja serpiente cornuda: una mirada desde Mesoamérica. Arqueología, 36,135-158.

Martínez-González, R., Viñas, R. y Mendoza, L. (2009). Cueva de la Serpiente. Los ofidios con cuernos en la iconografía rupestre de Mulegé, Baja California Sur, México. Arqueología, 40, 20-37.

Mata-Silva, V., Johnson, J. D., Wilson, L. D., \& García-Padilla, E. (2015). The herpetofauna of Oaxaca, Mexico: composition, physiographic distribution, and conservation status. Mesoamerican Herpetology, 2, 6-62.

McCue, M. D. (2006). Cost of producing venom in three North American pitviper species. Copeia, 4, 818-825.

Mendoza, R. G. (1977). Worldview and the monolithic temples of Malinalco, Mexico: Iconography and analogy in pre-columbian architecture. Journal de la Société des américanistes, 64, 63-80.

Morales-Mávil, J. E., Guzmán-Guzmán, S., Canseco-Márquez, L., Pérez-Higareda, G., González-Romero, A. y Vogt, R. C. (2011). La Biodiversidad en Veracruz: estudio de estado. México: CONABIO, Gobierno del Estado de Veracruz, Universidad Veracruzana, Instituto de Ecología A. C.

Morgenstern, D., \& King, G. F. (2013). The venom optimization hypothesis revisited. Toxicon, 63, 120-128.

Paredes-García, D. M., Ramírez-Bautista, A. y Martínez-Morales, M. A. (2011). Distribución y representatividad de las especies del género Crotalus en las áreas naturales protegidas de México. Revista Mexicana de Biodiversidad, 82, 689-700.

Rivas, M. E. A. (2014). Caracterización bioquímica del veneno de tres especies de serpientes de cascabel de montaña (Crotalus aquilus, C. lepidus klauberi y C. lepidus aquilus) (tesis de maestría). Aguascalientes: Universidad Autónoma de Aguascalientes.

Tay, Z. J., Díaz, S. J. G., Sánchez, V. J. T., Ruiz, S. D. y Castillo, L. (2002). Serpientes y reptiles de importancia médica en México. Revista de la Facultad de Medicina, UNAM, 45(5), 212-219.

Uetz, P. (2018). The reptile data base. Disponible en http://www.reptile-database.org/. Consultado el 27 de mayo de 2018.

Valencia-Aguilar, A., Cortés-Gómez, A. M., \& Ruiz-Agudelo, C. A. (2013). Ecosystem services provided by amphibians and reptiles in neotropical ecosystems. International Journal of Biodiversity Science, Ecosystem Services y Management, 9, 257-272.

White, J., Warrell, D., Eddleston, M., Currie, B. J., Whyte, I. M., \& Isbister, G. K. (2003). Clinical Toxicology-Where are we now? Journal of Toxicology: Clinical Toxicology, 41, 263-276.

Zertuche, J. J. (1981). Reptiles mexicanos de importancia para la salud pública y su distribución geográfica. $S a$ lud Pública de México, 23, 329-343. 